\title{
Three-Dimensional Simulation of Aggregate and Asphalt Mixture Using Parameterized Shape and Size Gradation ${ }^{1}$
}

\author{
Jue Li*, Junhui Zhang*, Guoping Qian*\&, Jianlong Zheng*, Yuqing Zhang ${ }^{\$}$ \\ *National Engineering Laboratory of Highway Maintenance Technology, Changsha University of Science \& \\ Technology, Changsha, China. \\ ${ }^{\$}$ Aston Institute of Materials Research, School of Engineering and Applied Science, Aston University, UK. \\ ${ }^{\&}$ corresponding author, Email: zjhseu@ csust.edu.cn
}

\begin{abstract}
Aggregate occupies at least three-quarters of the volume of asphalt mixture and can significantly affect the performance of pavement. The geometrical morphology influences the slippage and interlock among aggregates for resisting and distributing applied loads. In recent years, the discrete element method (DEM) has been employed for simulation of asphalt mixture structure. This paper introduces an approach for simulation of aggregate and asphalt mixture using parameterized shape and size gradation. Both plane geometry factor $(P G F)$ and section aspect ratio $(S A R)$ were employed to describe the 3D geometric characteristics of aggregates. A numerical technique of aggregate models was implemented with probabilistic parameters depending on statistical results of $P G F \mathrm{~s}$ and $S A R \mathrm{~s}$. Therefore, the 3D numerical model of asphalt mixtures was assembled with three different components, which is validated by uniaxial compression test via comparison with that of the laboratory result. It was found that the $P G F$ and $S A R$ are appropriate to describe the three-dimensional features of aggregate shapes, due to the fact that a simplified space object can be described by a 2D graphical projection and a vector scalar corresponding to the space vector. Probability distribution curves of $P G F \mathrm{~s}$ and $S A R$ s between coarse aggregates are in concordance with the Gauss-type function, since their correlation coefficients are all greater than $95 \%$. It was verified that the developed clumping algorithm of aggregates was reasonable with the shapes and size gradation. Based on the parallel-bond model and the Burger's model, the results of virtual tests are in good agreement with those of laboratory uniaxial tests. It is shown that the angularity $(P G F)$ of aggregates has a beneficial effect on the strength and stability while the flat-elongated feature $(S A R)$ has a negative effect on those of asphalt mixtures.
\end{abstract}

Author keywords: asphalt mixtures; irregular aggregates; three-dimensional simulation; discrete element method; parameterized shape

\section{Introduction}

Asphalt mixture is a heterogeneous material, whose mechanical properties are determined by the characteristics of its components including aggregates, asphalt mastic and air voids (Liu and You 2009; Chen et al. 2015a). It is wellknown that the geometrical morphology and distribution of the aggregates play critical roles in forming the skeleton of the material structure, while the properties of the asphalt binder affect the mixture interface behavior (Singh et al. 2014). The discrete element method (DEM) is an efficient computational method for predicting the structural and mechanical characteristics of the asphalt mixtures (You and Buttlar 2004). Over the past two decades, the DEM has been widely applied to analysis of the interface contact behavior between aggregates in the mixtures (Liu et al. 2011;

\footnotetext{
${ }^{1}$ This is an Accepted Manuscript of an article published by Journal of Materials in Civil Engineering (ASCE). The final
} publication is available online via https://ascelibrary.org/doi/abs/10.1061/\%28ASCE\%29MT.1943-5533.0002623 
Dan et al. 2018).

With the development of computer technology and DEM theory, the DEM models has evolved from two dimensions to three dimensions, in which three grain types are available to model granular materials including spherical particles, clusters and clumps (Zhang, et al. 2018a). The ideal particle system such as disk (2D) or spheres (3D), in general, is the basic calculating element of the DEM controlled by Newton's 2nd law and specific contact rules. It is deemed to be an effective way to simulate the anisotropic material through different rolling frictional resistances (Wensrich and Katterfeld 2012) and is more efficient than the other two types in terms of contact searching and force calculation (Ferellec and Mcdowell 2010). Some researchers suggested that the DEM model of spherical particles is capable of properly estimating mix designs of aggregate structures and the development trend in the compaction process of asphalt mixtures (Shen and Yu 2010; Chen et al. 2015b). However, these models cannot accurately evaluate the movement and rotation of granular materials (Liu and You 2011). Meanwhile, the angularity and complex shape of actual granules provide complete contact and interlocking, which affect the mechanical properties of stone-based materials under loading ( $\mathrm{Lv}$ et al. 2018). In this regard, the ideal particles such as spheres or ellipsoids cannot capture the effect (Shi et al. 2015). Both clusters and clumps were developed to represent the irregular and angular shape of natural structures, fully filled with overlapping or non-overlapping discrete elements, within the boundary of polyhedron or hyper quadric (Fu et al. 2010). A single cluster is made up of a series of bonded small disks (or spheres) through a default structural strength and can interact with the others, approximating the packing behavior of a blocky system (Zhang and Wong 2018d). Clumps also behave as rigid bodies but will never break apart, on account of the relative position of slaved particles fixed by the clump logic (Itasca Consulting Group 2005). Based on this logic mode, the efficiency of the time stepping in DEM has been improved significantly. The aggregates can be regarded as a set of clumps at loading routine, whose internal damage don't occur.

It is very important for asphalt mixture digital specimens to obtain and reconstruct heterogeneous models (Yang et al. 2016). Recently, many studies have been reported to develop reliable approaches to analyze and simulate coarse aggregate irregular attributes. In this issue, the clumping technology is widely used and can be divided into the following four processes. First, the digital image technology was applied to analyze different aggregate properties in 2D DEM model but limited to the plane (Mahmoud et al. 2010; Peng and Sun 2016). Next, the aggregate clumping templates were created in 3D by extending multiple 2D image features in order and the accuracy of aggregate models depends on the quality of the processed image (Yu and Shen 2012). Then, with the development of the digital image technology, the realistic aggregates can be reconstructed in DEM through some sophisticated imaging equipment, such as the X-ray CT system (Liu et al. 2017) and the 3D Sobel-Feldman operation (Yang et al. 2017). The main disadvantage of the digital image technology is that the acquired samples of aggregates are insufficient and unchanged in shape, due to current technical constraint and high experimental costs. Finally, the gradation distribution and the irregular shape of aggregates were expressed by the random mathematic model, which is considered to be an effective way to describe the mechanical heterogeneity of asphalt mixtures (Ma et al. 2017). The random mathematic model is efficient and unlimited but the impact of real aggregate characteristics is weakened relative to digital technology (Salemi and Wang 2018). In this study, both digital image technology and random mathematic model were taken into consideration to reconstruct numerical specimens of asphalt mixture.

The objective of this paper is to present an approach for parameterized characterizations of particle shapes and numerical reconstructions of asphalt mixtures. The characteristics of aggregates were obtained by digital image technology. Both the plane geometry factor $(P G F)$ and the section aspect ratio $(S A R)$ were employed to describe 3D geometric properties and analyze the compaction effects of aggregate shapes on the assembly. Moreover, a digital aggregate generation algorithm was proposed and its reliability was verified by testing volume distribution of coarse aggregates. Finally, the numerical specimen of asphalt mixtures was assembled with three different components, namely aggregates, mortar and voids. Meanwhile, the effect of PGFs and SARs on compressive strength was 
discussed.

\section{Gradations and attributes of asphalt concrete mixtures}

Three gradations of asphalt concrete (AC) mixture, namely AC-16, AC-20 and AC-25, were selected for the study, as shown in Fig.1. These types of asphalt concrete are applied to the representative structures of asphalt pavement in China, such as the middle and lower surface course. According to the Chinese flexible pavement design (CCCC Infrastructure Maintenance Group 2017), the coarse aggregate is required to be cubic-shaped with rough surfaces and its diameter must be greater than $4.75 \mathrm{~mm}$ (No. 4 sieve). The aggregates obtained from the margins of rivers can be used for low-volume road for cost efficient reason, but pebbles and other natural materials are not recommended for the use in asphalt mixtures of highways in China. Therefore, this study is focused mainly on the effect of cubicshaped characteristics of the aggregates on the properties of mixtures. And the crushed limestone aggregate was selected in the research and procured from quarries around Zhuzhou. By means of the Marshall tests, the optimum asphalt content of AC-16, AC-20 and AC-25 was determined to be 5.1\%, 4.6\% and 4.0\%, respectively.

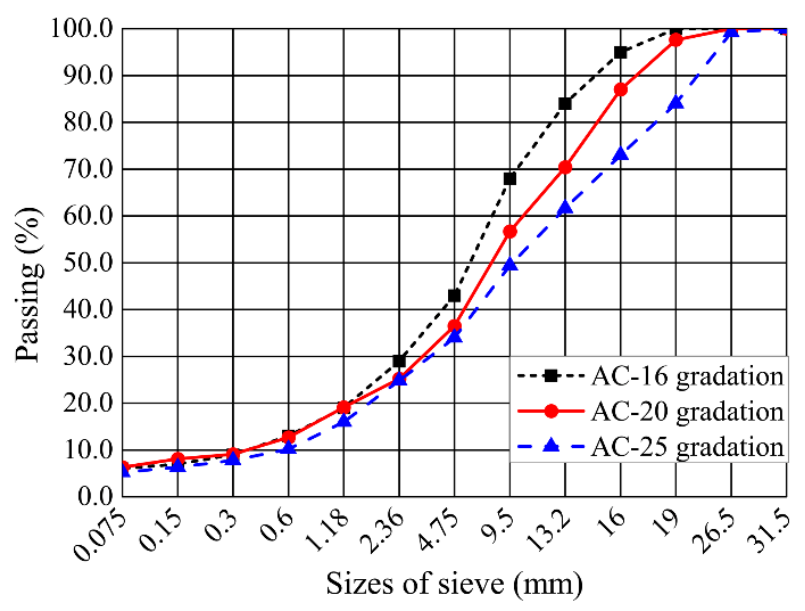

Figure 1: Gradations of Asphalt Concretes

\section{Three-dimensional description of aggregate shape}

The 3D object of aggregates is generally modeled by multiple 2D geometric outlines and fine-divided networks, but this approach demands excessive memory and computer time (Chen et al. 2017). Some simplified parameters have been proposed to describe the shape and texture of aggregates, so as to reduce costs and time associated with 3Dmodeling. Meanwhile, many researches indicated that these parameters have dramatic effects on mechanical properties of aggregates and asphalt mixtures.

In fact, a space object can be determined by a 2D graphical projection and a vector scalar corresponding to the space vector, as shown in Fig. 2. In this study, the digital image technology was applied to obtain overall sample characteristics of aggregates, such as the geometric shape of a projection plane, the height of a cross section and the maximum axis length. Two geometric parameters were employed to characterize the feature of aggregate particles in 3D. The plane geometry factor $(P G F)$ is defined as the edge number of the projection plane geometry of an aggregate. The section aspect ratio $(S A R)$ is calculated by the ratio of the maximum axis length to the cross-sectional height, in which the 3D roundness of the aggregate was considered. Details about the shape feature access and probability analysis of aggregate samples are discussed below. 


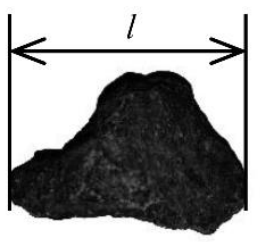

Front view

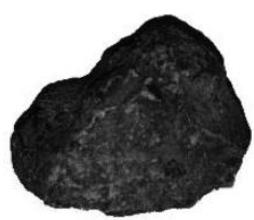

Pictorial diagram

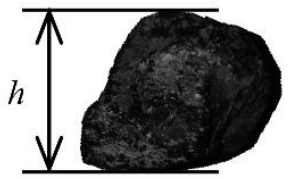

Right view

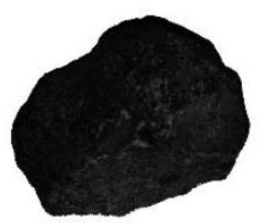

Top view

Figure 2: Digital image and projection of an aggregate

\section{Impact analysis of geometric parameters on the assembly}

The influences of $P G F \mathrm{~s}$ and $S A R$ s on the packing characteristics of aggregate mixtures were studied by measuring porosities in the biaxial and triaxial compression tests with a confining pressure of $10 \mathrm{MPa}$ (Zhang et al. 2018b; Zhang et al. 2018c). The initial specimens were $200 \mathrm{~mm}$ in height and $100 \mathrm{~mm}$ in diameter. According to the three grading distributions in Fig.1, disk (ball) particles and single shaped polygon (ellipsoid) clumps were tested separately for coarse aggregates. The elastic modulus of the crushed limestone is $39.3 \mathrm{GPa}$ and its Poisson's ratio is 0.23 on average (Wang 2011).

The Bubble Pack algorithm was mentioned to generate clumps using an input closed geometry (line or triangular surface) (Taghavi 2011). Default values were used in the algorithm for the ratio of the smallest to largest ball in the clump which is 0.5 and the angular corresponding to smoothness which is $150^{\circ}$ for this. It has demonstrated that these parameters have little effect on the corresponding geometry of the clumps (Liu et al. 2017) and the optimized solution was not included in this research. As show in Fig.3, the surface descriptor of the clump was imported and the ellipsoid clumps in 3D with different SARs (1.5, 2.0, 2.5, 3.0 and 3.5) were established. Similarly, Fig.4 shows that disk clumps with different $P G F$ s $(4,5,6,7$ and 8$)$ were imported by regular polygons.

\begin{tabular}{|c|c|c|c|}
\hline SARs & $\begin{array}{c}\text { Number } \\
\text { of balls }\end{array}$ & Geometries & Clumps \\
\hline 1.5 & 3 & & \\
\hline 2.0 & 6 & & \\
\hline 2.5 & 8 & & \\
\hline 3.0 & 9 & & \\
\hline 3.5 & 11 & & \\
\hline
\end{tabular}

Figure 3: Clumps of ellipsoid geometry with different SARs 


\begin{tabular}{|c|c|c|c|}
\hline PGFs & $\begin{array}{c}\text { Number } \\
\text { of balls }\end{array}$ & Geometries & Clumps \\
\hline 4 & 25 & & \\
\hline 5 & 21 & \\
\hline 6 & 19 & \\
\hline 7 & 22 & \\
\hline 8 & 17 & \\
\hline
\end{tabular}

Figure 4: Clumps of regular polygon with different $P G F \mathrm{~s}$

The test results of polygon (ellipsoid) clumps with different $S A R$ s and $P G F$ s are shown severally in Fig.5 and Fig.6. For biaxial tests, the disk particles ( $P G F$ is similar to infinity, marked as ' $\infty$ ') were replaced in turn by a set of the same shape regular polygon. It is clearly that the $P G F$ has an effect on the final porosity and its trend is not monotonic. The reason for this issue may be that the roundness of particles will increase and the interlock between particles will be weakened after the $P G F$ reaches a certain value. For triaxial tests, the porosity increases with the $S A R$ and the compaction effect of balls is different from the ellipsoids. Through five parallel experiments, the error bars represent the variation of results. At the same confining pressure, the porosity depends on the internal structure and contacts. On these results of regular polygons and ellipsoids, it is noted that both shape and gradation affect the composition of internal structure and the contact behavior between particles.

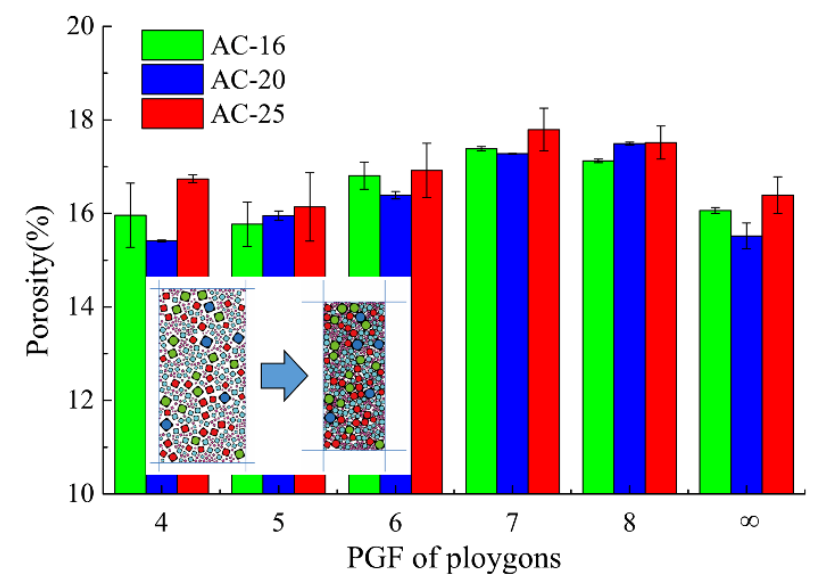

Figure 5: Final porosity of biaxial compression tests 


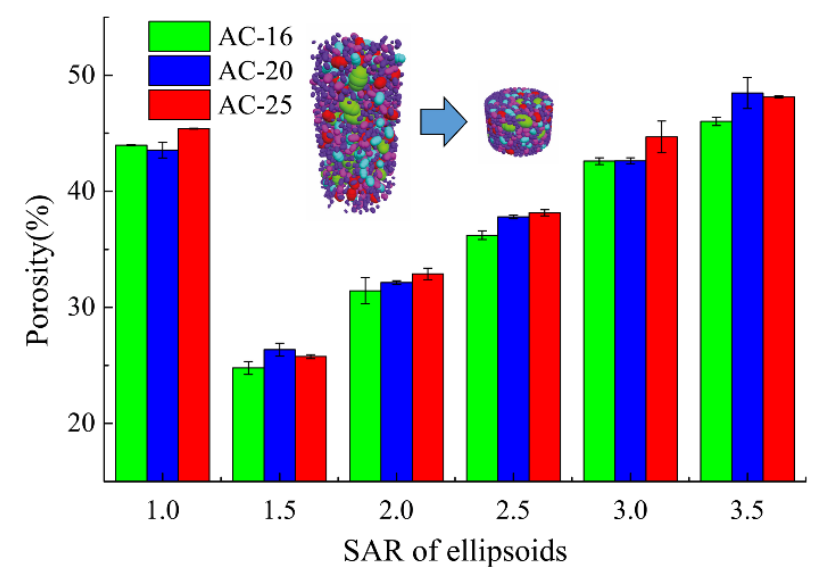

Figure 6: Final porosity of triaxial compression tests

\section{Investigation and data collection of real aggregates}

Two-dimensional images of aggregates were photographed to identify the projected surface features of aggregates using a high-definition camera. The grayscale images were obtained by the gray scale enhancement and de-noise processing, as shown in Fig.7. Since the maximum nominal particle diameters of AC-25 mixture is $26.5 \mathrm{~mm}$, the coarse aggregates in this research are from six sieve size groups $(26.5 \mathrm{~mm}-31.5 \mathrm{~mm}, 19 \mathrm{~mm}-26.5 \mathrm{~mm}, 16 \mathrm{~mm}-19 \mathrm{~mm}$, $13.2 \mathrm{~mm}-16 \mathrm{~mm}, 9.5 \mathrm{~mm}-13.2 \mathrm{~mm}, 4.75 \mathrm{~mm}-9.5 \mathrm{~mm})$. The object of this study is a cylinder specimen of asphalt mixtures, including hundreds of coarse aggregates for each size group. The measured aggregates were randomly selected by the cluster sampling and numerical features of the aggregates were given by the Monte-Carlo method (Ferellec and Mcdowell 2010). Through multiple sampling analysis, the variance of statistical results tends to stabilize within $10 \%$ relative error when the number of particles is great than 100 . To represent the geometric feature of a whole specimen, the sample size per sieve was set to be 200. Due to little demand for large-size aggregates, the number of aggregates at first sieve was 100 .

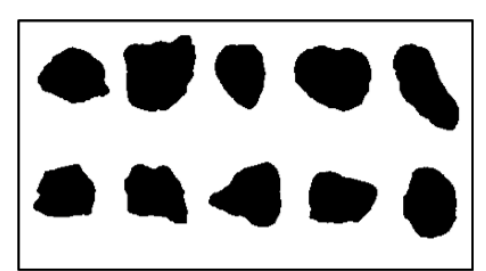

(a)

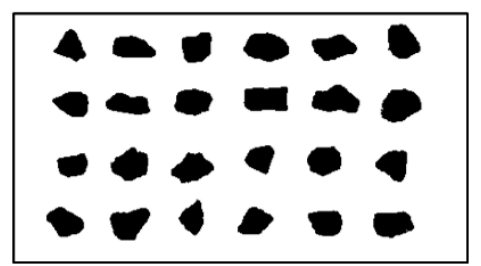

(d)

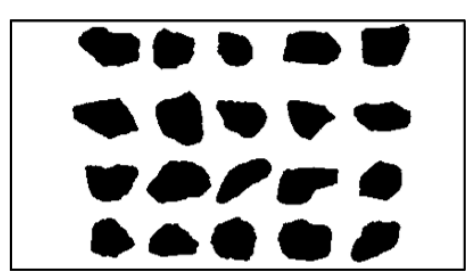

(b)

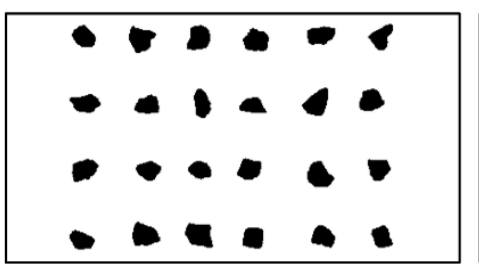

(e)

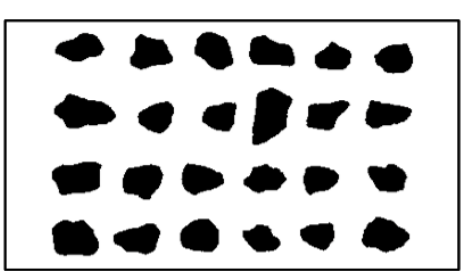

(c)

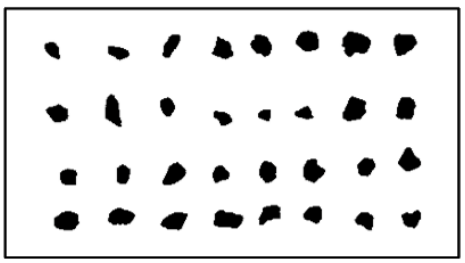

(f)

Sieve size (mm): a- 26.5 ; b- 19.0; c- 16.0 ; d- 13.2; e- 9.5; f -4.75

Figure 7: Grayscale image of aggregate projection plane

The statistical distribution result of $P G F \mathrm{~s}$ and $S A R \mathrm{~s}$ is shown in Fig.8. A modified Douglas-Peucker algorithm was written in MATLAB that the thresholds were obtained by iterating over iterations to reduce the impact of special pixels and the polygon area was used to replace the nonlinear boundary curve of aggregate images (Visvalingam and 
Whyatt 1990). By means of polygonal fitting, the $P G F$ of aggregates per sieve was counted and the results show that a peak value occurs as the $P G F$ increases. The probabilities for the $P G F$ of 5 and 6 are both greater than $30 \%$ in the same sieve. Probability distribution curves of $P G F \mathrm{~s}$ and $S A R \mathrm{~s}$ are in concordance with the Gauss-type function. With the increase of $S A R \mathrm{~s}$, the trend of probability distribution is similar to that for $P G F \mathrm{~s}$. Meanwhile, the statistical analyses indicate that the aggregate size has a significant influence on the statistical results of SARs but the difference is negligible on those of $P G F \mathrm{~s}$.
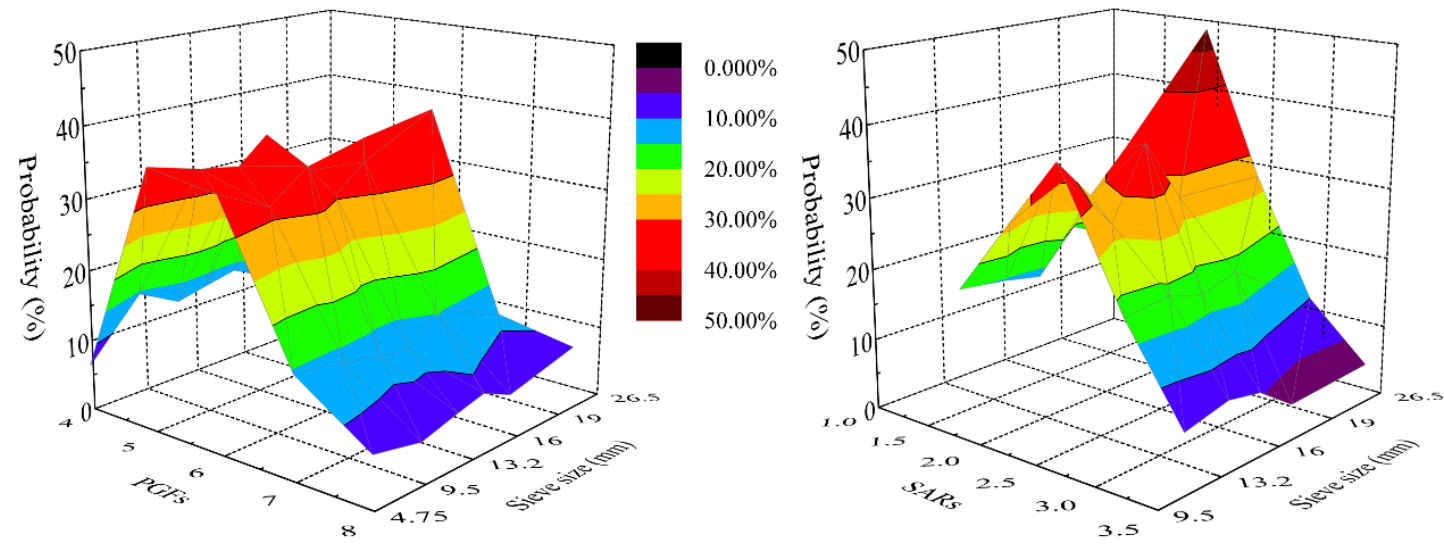

Figure 8: Statistical distribution results of $P G F \mathrm{~s}$ and $S A R \mathrm{~s}$

Gaussian fitting functions and parameters of $P G F \mathrm{~s}$ and $S A R$ s are displayed in Table 1 and Table 2, respectively. For the peak feature of $P G F \mathrm{~s}$, the mean value $x_{c}$ is from 5.393 to 5.577 , which is within the range at probability of $30 \%$. The mean value for SARs is from 1.858 to 2.099 and the peak of statistical results is also around 2.0. The correlation coefficients of fitting curves, $R$ values, are all greater than $95 \%$. It can be assumed that Gaussion fitting curves are in good agreement with the statistical results of aggregates per sieve. Therefore, these fitting parameters were applied to represent the geometry information of the realistic aggregates in mathematical models.

Table 1. Gaussian curve fitting results of $P G F \mathrm{~s}$

\begin{tabular}{cccccc}
\hline \multirow{2}{*}{$\begin{array}{c}\text { Sieve size } \\
(\mathrm{mm})\end{array}$} & \multicolumn{4}{c}{$y=y_{0}+\frac{A}{w \sqrt{\pi / 2}} e^{-2 \frac{\left(x-x_{c}\right)^{2}}{w^{2}}}$} & $R$ \\
\cline { 2 - 5 } & $y_{0}$ & $x_{c}$ & $w$ & $A$ & \\
\hline 26.5 & 0.084 & 5.577 & 1.160 & 0.584 & 0.998 \\
19.0 & 0.057 & 5.585 & 1.929 & 0.743 & 0.999 \\
16.0 & 0.093 & 5.393 & 1.365 & 0.557 & 0.999 \\
13.2 & 0.080 & 5.532 & 1.445 & 0.623 & 1.000 \\
9.5 & 0.053 & 5.436 & 1.878 & 0.766 & 0.999 \\
4.75 & 0.080 & 5.498 & 1.279 & 0.609 & 0.981 \\
\hline
\end{tabular}

Table 2. Gaussian curve fitting results of $S A R \mathrm{~s}$

\begin{tabular}{cccccc}
\hline \multirow{2}{*}{$\begin{array}{c}\text { Sieve size } \\
(\mathrm{mm})\end{array}$} & \multicolumn{4}{c}{$y=y_{0}{ }^{\prime}+\frac{A^{\prime}}{w^{\prime} \sqrt{\pi / 2}} e^{-2 \frac{\left(x^{\prime}-x_{c}^{\prime}\right)^{2}}{w^{\prime 2}}}$} & $R$ \\
\cline { 2 - 5 } & $y_{0}{ }^{\prime}$ & $x_{c}{ }^{\prime}$ & $w^{\prime}$ & $A^{\prime}$ & \\
\hline 26.5 & 0.049 & 1.858 & 0.670 & 0.378 & 0.991 \\
19.0 & -0.235 & 1.955 & 2.077 & 1.450 & 0.990 \\
16.0 & 0.055 & 2.099 & 0.941 & 0.376 & 0.991 \\
13.2 & 0.043 & 1.989 & 1.167 & 0.420 & 0.960 \\
9.5 & -0.004 & 2.076 & 1.255 & 0.542 & 0.987 \\
\hline
\end{tabular}




\section{An aggregate generation algorithm with geometric parameters}

According to statistic information of geometrical features, an aggregate generation algorithm was developed by the Monte-Carlo method. The model of asphalt mixture structures was divided into three components: coarse aggregate, asphalt mortar and void. Limited by computer memory, the following discussion is only for coarse aggregate (> $4.75 \mathrm{~mm})$.

\section{Clumping model of irregular aggregates}

The irregular clumps were processed through a newly developed aggregate generation algorithm to simulate the real aggregates with the Gauss parameters. Three steps were mentioned as follows.

1) The first step is to create the basic clump models.

The spatial structure of an aggregate is a cuboid inscribed in a certain sphere, with a square bottom whose edge length $l_{\mathrm{b}}$ was determined by the uniform distribution of the aggregate size and the clump element radius, using Eq. (1).

$$
l_{\mathrm{b}}=\operatorname{int}\left(\frac{\sqrt{2}\left(R_{\min }-\left(R_{\max }-R_{\min }\right) \times \operatorname{urand}()\right)}{r_{\mathrm{e}}}\right) \times r_{\mathrm{e}}
$$

where int() is the integer function; urand() is the uniformly distributed random number from 0 to $1.0 ; R_{\max }$ and $R_{\min }$ are the maximum and the minimum aggregate size in the sieve; $r_{\mathrm{e}}$ is the clump element radius.

The height of the cuboid $h_{\mathrm{c}}$ could be expressed by the $S A R$, as shown in Eq. (2). Clump elements were stacked in a rectangular arrangement with a spherical distance of the radius and the diagram of clump elements was shown in Fig.9.

$$
h_{\mathrm{c}}=\operatorname{int}\left(\frac{\sqrt{2} l_{\mathrm{b}}}{\operatorname{grand}\left(y_{0}, A, x_{c}, w^{2}\right) \times r_{\mathrm{e}}}\right) \times r_{\mathrm{e}}
$$

where grand() is the Gauss distribution random number from 1.0 to $3.5 ; y_{0}, A, x_{\mathrm{c}}$ and $r_{\mathrm{c}}$ are the fitting parameters of the $S A R$.

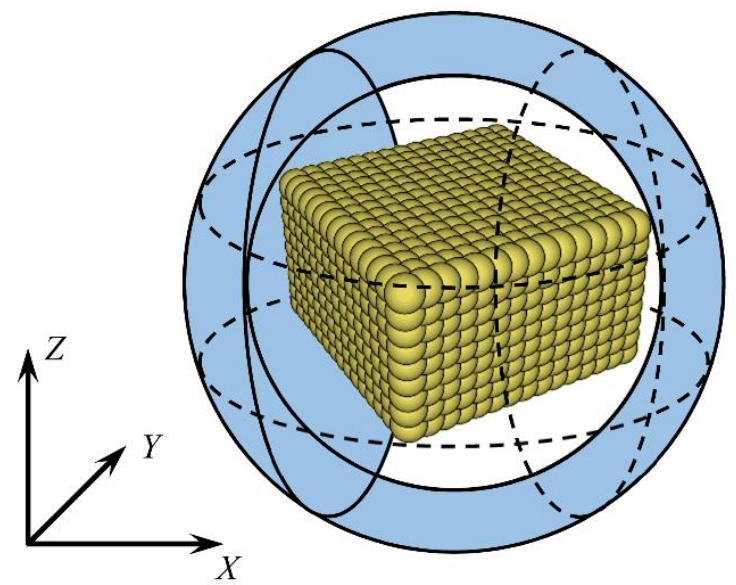

Figure 9: Elements accumulation of basic clump model

A judgment mechanism was required to ensure that the clump size conforms to the grading requirements, as described in Eq. (3). If the conditions were not met, the above steps would be repeated to regenerate the stochastic model.

$$
R_{\min }^{2}<l_{\mathrm{b}}^{2}+h_{\mathrm{c}}^{2}<R_{\max }^{2}
$$

2) The second step is to segment the plane of the basic model. 
For the model in line with the aggregate edge characteristics, a random plane was set by a FISH function to incise the basic model to obtain a complex aggregate space structure, as shown in Fig.10. The plane was through a specific point in the inscribed sphere and the direction of the outside line was the body-center to the point, as expressed in Eq. (4).

$$
\left(x_{1}-x_{0}\right)\left(x-x_{1}\right)+\left(y_{1}-y_{0}\right)\left(y-y_{1}\right)+\left(z_{1}-z_{0}\right)\left(z-z_{1}\right)=0
$$

where $x_{0}, y_{0}$ and $z_{0}$ are the coordinate of the clump body-center; $x_{1}, y_{1}$ and $z_{1}$ are the coordinate of the specific point in an inscribed sphere.

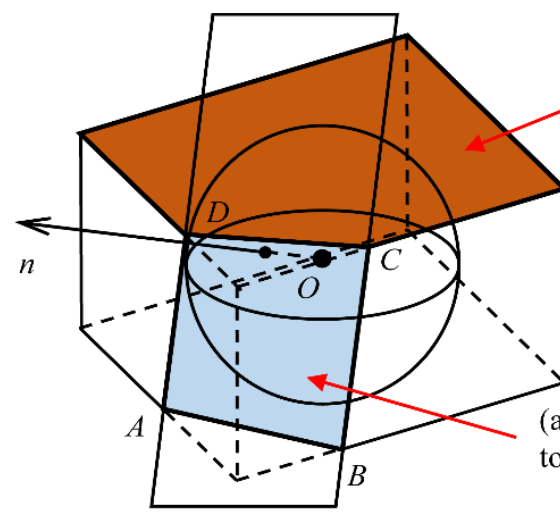

(b) Pentagonal surface by plane cutting

Figure 10: Schematic diagram of polygon segmentation by plane

The position of the specific point must meet the requirement of Eq. (5), to ensure the effectiveness of incision.

$$
\begin{aligned}
& z_{1}=z_{0}+0.5 \times \frac{h_{\mathrm{c}}{ }^{2}}{l_{\mathrm{b}}} \operatorname{urand}() \\
& y_{1}=y_{0}+0.5 h_{\mathrm{c}} \sin (2 \pi \theta) \\
& x_{1}=x_{0}+0.5 h_{c} \cos (2 \pi \theta)
\end{aligned}
$$

where $\theta$ is the uniformly distributed random number from 0 to 1.0 .

3) The last step is to obtain the irregular clump with polygon bottom.

The clumping model was cut into any polygon shape in the bottom by repeating the second step. Probabilistic fitting functions of the $P G F$ were also applied to determine the polygon of clump bottoms by the number of cutting planes randomly. Fig.11 shows the typical digital models of aggregate particles in each sieve.

\begin{tabular}{|c|c|c|c|c|}
\hline Size(mm) & \multicolumn{5}{|c|}{ Clumps of aggregate } \\
\hline 26.5 & & & & \\
\hline 19 & & & & \\
\hline 16 & & & & \\
\hline 13.2 & & & & \\
\hline 9.6 & & & & \\
\hline 4.75 & & & & \\
\hline
\end{tabular}

Figure 11: Typical digital aggregates with each sieves 


\section{Aggregate accumulation with the size gradation}

During the grading delivery process of the above-mentioned digital random aggregate, two issues need to be solved: 1) how to determine the location of a random aggregate in a specified space; 2) how to achieve the aggregate quality distribution as grading scheme.

Fig.12 shows the flow diagram of the grading delivery algorithm. The command GEN in PFC3D was used to randomly generate ball particles in the specified area and balls of different particle sizes could be dispensed in sieve $i$. The balls were derived with the coordinates and replaced by random aggregate clumps. At the same time, the total mass of the generated aggregate clumps was computed to determine whether the set quality reached. If the desired result is not obtained, the above procedures should be repeated to generate more balls and clumps.

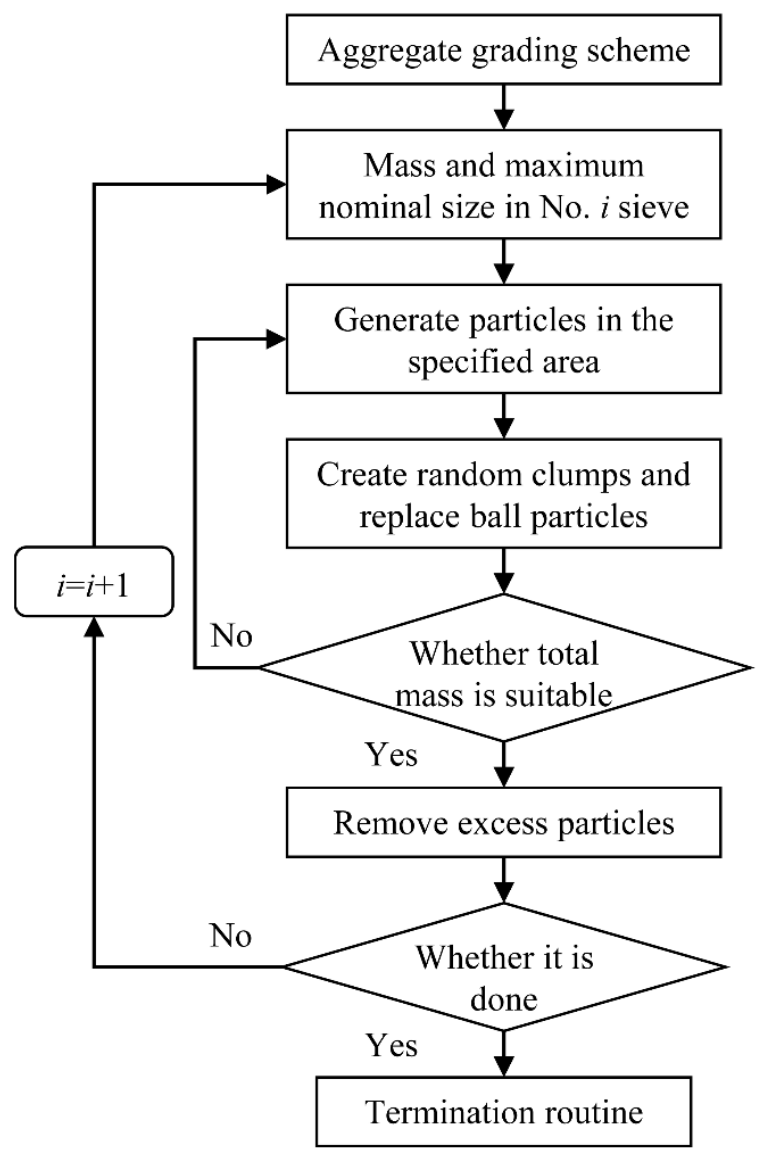

Figure 12: Flow diagram of the grading delivery algorithm

\section{Verification of volume distribution in aggregate skeleton}

In general, it is possible to change the nominal maximum aggregate size when the cuboid is cut into different shapes. A geometrical constraint was defined to reduce the possibility in this process. Both the maximum diagonal length of the bottom surface and the height of aggregates were obtained and the quadratic sum must be greater than the size of their primary control sieve. In addition, the size of the final aggregate has less effect on the whole gradation since there is a large difference between each sieve size.

Taking the assembly of AC-20 mixture as an example, the correlation between designed and generated gradation was analyzed by volume content of aggregates, as shown in Fig.13. Aggregate clumps were divided into 5 groups according to the grain diameter and their volume can be calculated by the number of assigned particles. To understand the stability of the model, multiple numerical modeling tests were conducted to measure the variation of volume fraction per aggregate group. The results show that the volume distribution of generated aggregates is consistent with 
the design gradation due to $R^{2}$ of $99.45 \%$. Therefore, it is proved that the developed aggregate generation algorithm is reasonable and stable in this research and can be applied to the follow-up modeling of asphalt mixtures.

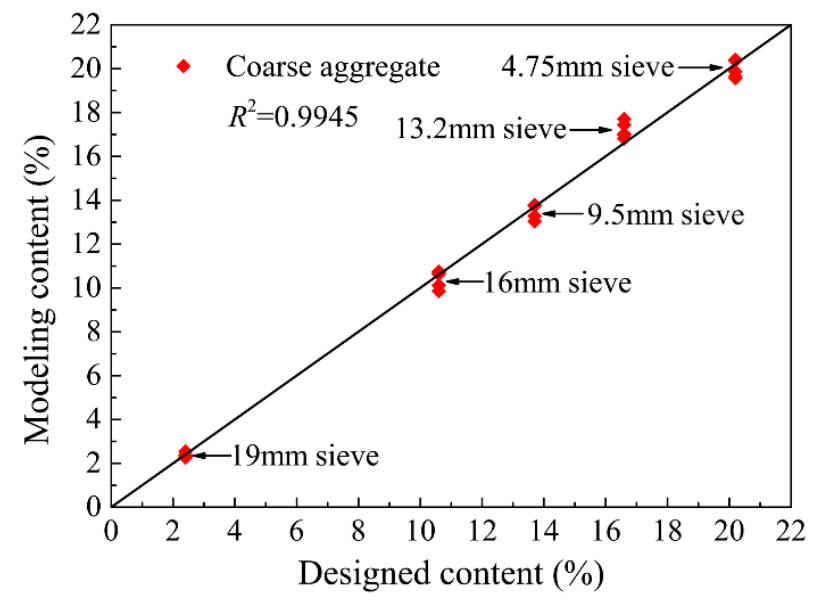

Figure 13: Correlation between designed and generated gradation

\section{Simulation and analysis of asphalt mixtures using uniaxial tests}

\section{Numerical specimen of asphalt mixture}

The three-dimensional cylindrical specimen of asphalt mixtures was built with the size of $100 \mathrm{~mm}$ in height and $100 \mathrm{~mm}$ in diameter. The coarse aggregates were delivered and the initial height was determined to be $250 \mathrm{~mm}$ by multiple testing to ensure the produced particles enough. After the coarse aggregate had been finished, the upper wall was controlled to compress the specimen until the height was reduced to $100 \mathrm{~mm}$ and the assembly tended to balance through manifold cycles, as shown in Fig.14 (a).

Due to the current technical difficulties, the coarse aggregate skeleton is not able to be filled with dense mortar particles. To obtain the dense structure of asphalt mixtures, a simplified approach to fill the asphalt mortar was proposed based on coarse aggregate skeleton. A numerical specimen of asphalt mixtures was reconstructed by singlesized particles with $2.5 \mathrm{~mm}$ in diameter and a total number of 49520, as shown in Fig.14 (b). A FISH function was written to determine whether the filled particles overlapped with the original aggregate clumps, which were pregenerated by the aggregate generation algorithm. It is clearly that the single-sized particles are colored to represent different components of asphalt mixtures in Fig.14 (c). If the overlap was present, it would be defined as the aggregate unit (dark-colored part); otherwise, it would be defined as the mortar unit (light-colored part). It is noted that these aggregates are still a series of irregularly-shaped clumps, whose ranges are consistent with the original aggregate skeleton. Similarly, some mortar particles were randomly removed to simulate the void distribution. The nominal porosity is 0.04 in this model.

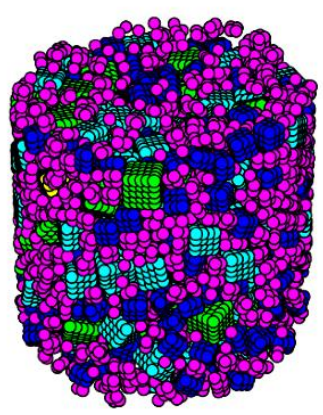

(a) Aggregate skeleton

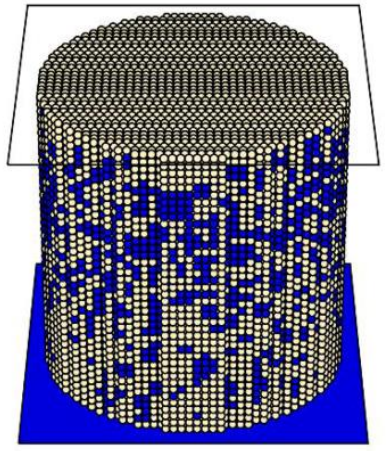

(b) Specimen by single-sized particles

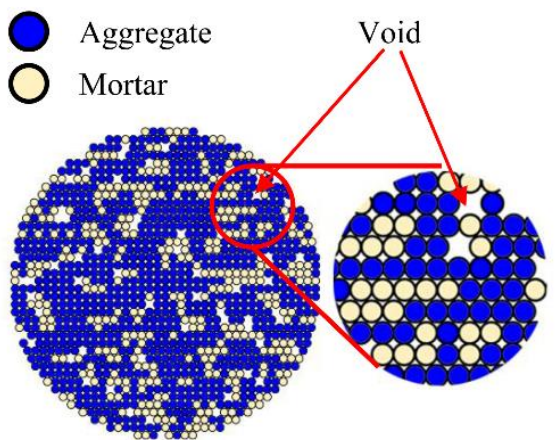

(c) Components of asphalt mixture

Figure 14: Digital specimen of asphalt mixtures 


\section{Contact model and material parameter}

Since the aggregates and the asphalt mortar have different mechanical properties, the components of the asphalt mixtures should be given different contact models and parameters. The aggregate-in interface was fixed by the clump definition; the aggregate-aggregate interface was defined as the slip model; the aggregate-mortar and the mortar-in interface were both defined as the parallel-bond model and the Burger's model.

The basic physical and mechanical properties of aggregates and asphalt mortar were measured by the corresponding mechanical test methods. The elastic modulus of the calcareous aggregate was 39.3GPa and Poisson's ratio was 0.23 (Wang 2011). The elastic modulus of the asphalt mortar was determined as $0.63 \mathrm{GPa}$ by laboratory tests and Poisson's ratio was 0.4. The Burger's model is used to characterize the viscoelastic behavior of materials and the parameters of this contact model were calculated by macroscopic mechanical parameters of creep behaviors (Itasca Consulting Group 2005). Therefore, the Burger's meso-mechanical parameters of the asphalt mortar at $20^{\circ} \mathrm{C}$ were obtained by the shear creep tests in laboratory and described as Table 3.

Table 3. Meso-mechanical parameters of Burger's model

\begin{tabular}{ccccc}
\hline Parameters & $C_{\mathrm{m}}$ & $K_{\mathrm{m}}$ & $C_{\mathrm{k}}$ & $K_{\mathrm{k}}$ \\
\hline Normal & $8.431 \times 10^{3}$ & $11.44 \times 10^{3}$ & $10.35 \times 10^{3}$ & $2.921 \times 10^{3}$ \\
Tangential & $3.01 \times 10^{3}$ & $4.09 \times 10^{3}$ & $3.70 \times 10^{3}$ & $1.04 \times 10^{3}$ \\
\hline
\end{tabular}

The uniaxial compression test was conducted by applying a constant rate of $2 \mathrm{~mm} / \mathrm{min}$ to the upper wall. The process of simulation was concluded when the stress was reduced to $80 \%$ of its historical peak, displayed in Fig. 15 . The parameters of the parallel-bond model have no definite correspondence with macroscopic mechanical behavior. Through multiple simulations and verification, the normal strength and shear strength of the parallel-bond model were determined to be $1.21 \times 10^{6}$ and $0.49 \times 10^{6}$, respectively. Fig. 15 shows that the results of virtual tests are in good agreement with those of laboratory tests in peaks and their curve slopes are very close from the initial state to the failure peak.

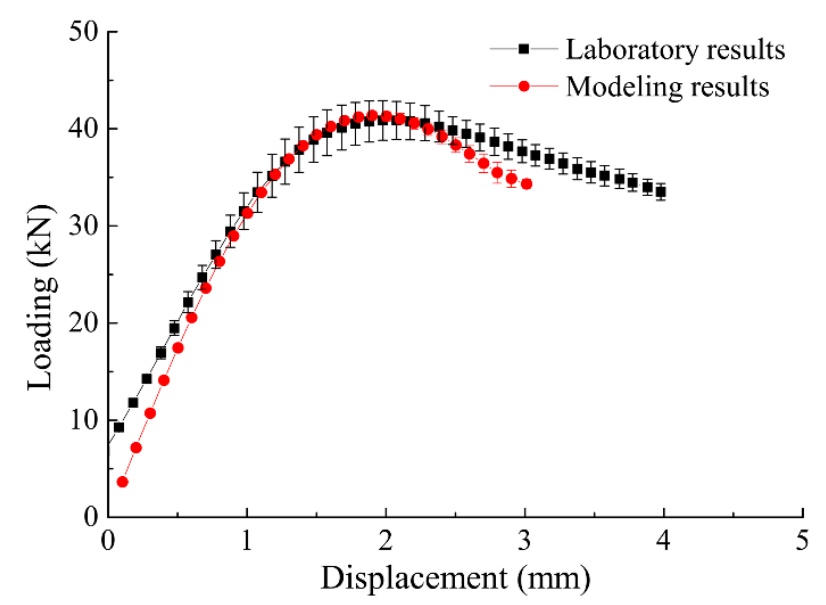

Figure 15: Results of uniaxial compression test in AC-20

\section{Impact analysis of PGFs and SARs on uniaxial tests}

It was mentioned in the previous work that the shape of aggregates plays an essential role in the mesoscopic structure of mixtures, such as coordination number and porosity. In addition, cubic-shaped aggregates with rough surfaces provide a more stable structure for the asphalt mixtures because those characteristics result in a better aggregate interlock. Thereby, a series of virtual uniaxial tests of asphalt mixtures were performed to understand the variation and its influencing factors of compressive strength.

Considering single dependence on PGFs and SARs, modeling test results are shown in Fig.16. The type of asphalt concrete makes a difference to mechanical properties. With the decrease of the maximum aggregate size, the 
compressive strength of all specimens increases. Meanwhile, as the PGF increases from 5.0 to 7.0, the strength increases greatly. However, it is obvious that the mechanical property is negatively correlated with SARs from 1.75 to 3.0. Thus, it is proved by simulations that the angularity $(P G F)$ of aggregates has a beneficial effect on the strength and stability while the flat-elongated feature $(S A R)$ has a negative effect on those of asphalt mixtures, which matches the real result.
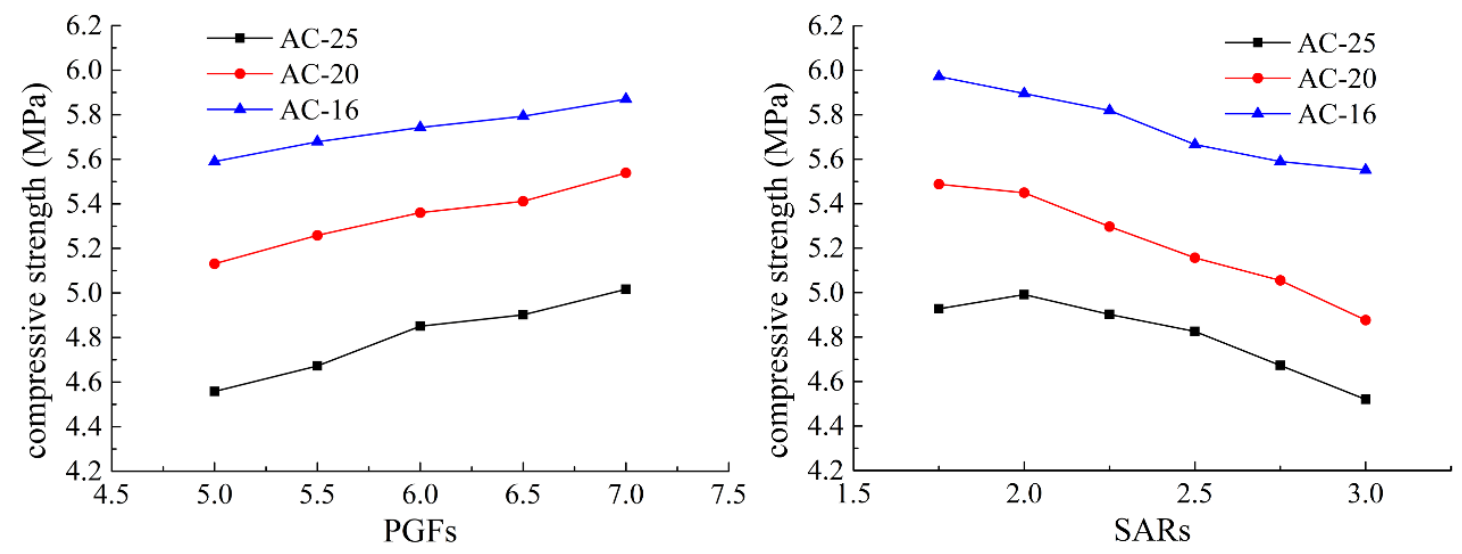

Figure 16: Effect of $P G F \mathrm{~s}$ and $S A R$ s on compressive strength

\section{Summary and conclusions}

In this study, the plane geometry factor $(P G F)$ and section aspect ratio $(S A R)$ were proposed to characterize the threedimensional structures of aggregates. A clumping model of irregular aggregates was developed and its input parameters were obtained through Gauss regression analysis of shape statistical results. A digital specimen of asphalt mixture was built with 49520 particles in 3D DEM. The uniaxial compression test was simulated and the effect of $P G F$ s and SARs on compressive strength was discussed. Major conclusions are drawn as follows:

1) Since a simplified space object can be described by a 2D graphical projection and a vector scalar corresponding to the space vector, the $P G F$ and $S A R$ are appropriate to represent the three-dimensional features of aggregate shapes. It was proved by biaxial and triaxial tests that both $P G F \mathrm{~s}$ and $S A R$ s play essential roles in the compaction property of aggregate mixtures.

2) Probability distribution curves of $P G F \mathrm{~s}$ and $S A R \mathrm{~s}$ are in concordance with the Gauss-type function. Their correlation coefficients of Gaussian fitting curves, $R$ values, are all greater than 95\%. Meanwhile, the statistical analyses indicate that the aggregate size has a significant influence on the statistical results of SARs but the difference is not observable on those of $P G F \mathrm{~s}$.

3 ) The developed clumping model of aggregates was reasonable with the shapes and size gradation. The final size of aggregates per sieve is strictly constrained and must be greater than the size of their primary control sieve. Meanwhile, it is verified that the volume distribution of generated aggregates is consistent with the design gradation due to $R^{2}$ of $99.45 \%$, taking AC-20 gradation as an example.

4) A cylinder specimen of asphalt mixtures can be reconstructed regularly by single-sized particles to distinguish three different components, namely aggregates, mortar and voids. Based on the parallel-bond model and the Burger's model, the results of virtual tests are in good agreement with those of laboratory uniaxial tests in peaks and their curve slopes are very close from the initial state to the failure peak. It is shown that the angularity $(P G F)$ of aggregates has a beneficial effect on the strength and stability while the flat-elongated feature $(S A R)$ has a negative effect on those of asphalt mixtures.

\section{Acknowledgements}


The authors gratefully acknowledge the National Key Research and Development Program of China (2017YFC0805307), National Natural Science Foundation of China (51478054, 51778071, 51878078), Excellent Youth Foundation of Natural Science Foundation of Hunan Province (2018JJ1026), Key Project of Education Department of Hunan Province (17A008). And it was supported by the Open Research Fund of Science and Technology Innovation Platform of State Engineering Laboratory of Highway Maintenance Technology, Changsha University of Science \& Technology (kfj150103).

\section{Conflict of Interest}

There is no conflict of interest.

\section{References}

CCCC Infrastructure Maintenance Group Co. Ltd. (2017). "Specifications for design of highway asphalt pavement." JTG D50-2017, China Communications Press, Beijing, China.

Chen, J., Zhang, M., Wang, H. and Li, L. (2015a). "Evaluation of thermal conductivity of asphalt concrete with heterogeneous microstructure." Appl. Therm. Eng., 84, 368-374.

Chen, J., Huang, B., Shu, X. and Hu, C. (2015b). "DEM Simulation of Laboratory Compaction of Asphalt Mixtures Using Open Source Code." J. Mater. Civil Eng., 27, 04014130.

Chen, J., Wang, H. and Li, L. (2017). "Virtual testing of asphalt mixture with two-dimensional and three-dimensional random aggregate structures." Int. J. Pavement Eng., 18(9), 824-836.

Dan, H. C., Zhang, Z., Chen, J. Q. and Wang, H. (2018). "Numerical simulation of an indirect tensile test for asphalt mixtures using discrete element method software." J. Mater. Civil Eng., 30(5), 04018067.

Ferellec, J. F. and Mcdowell, G. R. (2010). "A method to model realistic particle shape and inertia in DEM." Granul. Matter, 12(5), 459-467.

Fu, Y., Wang, L. and Zhou, C. (2010). "3D clustering DEM simulation and non-invasive experimental verification of shear localisation in irregular particle assemblies." Int. J. Pavement Eng., 11(5), 355-365.

Itasca Consulting Group, Inc. (2005). "PFC3D Version 3.1 Manual." Itasca Consulting Group, Inc., Minneapolis, Minnesota.

Liu, Y. and You, Z. (2009). "Visualization and Simulation of Asphalt Concrete with Randomly Generated ThreeDimensional Models." J. Comput. Civil Eng., 23(6), 340-347.

Liu, Y. and You, Z. (2011). "Discrete-element modeling: impacts of aggregate sphericity, orientation, and angularity on creep stiffness of idealized asphalt mixtures." J. Eng. Mech., 137, 294-303.

Liu, Y., Zhou, X., You, Z., Yao, S., Gong, F. and Wang, H. (2017). "Discrete element modeling of realistic particle shapes in stone-based mixtures through MATLAB-based imaging process." Constr. Build. Mater., 143, 169-178.

Lv, S., Liu, C., Chen, D., Zheng, J., You, Z. and You, L. (2018). "Normalization of fatigue characteristics for asphalt mixtures under different stress states." Constr. Build. Mater., 177, 33-42.

Ma, T., Wang, H., Zhang, D. and Zhang, Y. (2017). "Heterogeneity effect of mechanical property on creep behavior of asphalt mixture based on micromechanical modeling and virtual creep test." Mech. Mater., 104, 49-59.

Mahmoud, E., Masad, E. and Nazarian, S. (2010). "Discrete Element Analysis of the Influences of Aggregate Properties and Internal Structure on Fracture in Asphalt Mixtures." J. Mater. Civil Eng., 22(1), 10-20.

Peng, Y. and Sun, L. J. (2016). "Micromechanics-Based Analysis of the Effect of Aggregate Homogeneity on the Uniaxial Penetration Test of Asphalt Mixtures." J. Mater. Civil Eng., 28(11), 04016119.

Shen, S. and Yu, H. (2010). "Analysis of aggregate gradation and packing for easy estimation of hot-mix-asphalt voids in 
mineral aggregate." J. Mater. Civil Eng., 23(5), 664-672.

Shi, C., Li, D. J., Xu, W. Y. and Wang, R. (2015). "Discrete element cluster modeling of complex mesoscopic particles for use with the particle flow code method." Granul. Matter, 17(3), 1-11.

Singh, M., Kumar, P. and Maurya, M. R. (2014). "Effect of aggregate types on the performance of neat and EVA-modified asphalt mixtures." Int. J. Pavement Eng., 15(2), 163-173.

Salemi, M. and Wang, H. (2018). "Image-aided random aggregate packing for computational modeling of asphalt concrete microstructure." Constr. Build. Mater., 177, 467-476.

Taghavi, R. "Automatic clump generation based on mid-surface." Proc., Proceedings, 2nd International FLAC/DEM symposium, Melbourne, 791-797.

Visvalingam, M. and Whyatt, J. (1990). "The Douglas-Peucker algorithm for line simplification: re-evaluation through visualization." Computer Graphics Forum, Elsevier North-Holland, Inc, 213-228.

Wang, L. (2011). "Mechanics of asphalt: microstructure and micromechanics." McGraw Hill, New York.

Wensrich, C. M. and Katterfeld, A. (2012). "Rolling friction as a technique for modelling particle shape in DEM." Powder Technol., 217(2), 409-417.

Yang, X., You, Z., Wang, Z. and Dai, Q. (2016). "Review on heterogeneous model reconstruction of stone-based composites in numerical simulation." Constr. Build. Mater., 117, 229-243.

Yang, X., Chen, S. and You, Z. (2017). "3D Voxel-Based Approach to Quantify Aggregate Angularity and Surface Texture." J. Mater. Civil Eng., 29(7), 04017031.

You, Z. and Buttlar, W. (2004). "Discrete Element Modeling to Predict the Modulus of Asphalt Concrete Mixtures." J. Mater. Civil Eng., 16(2), 140-146.

$\mathrm{Yu}, \mathrm{H}$. and Shen, S. (2012). "Impact of aggregate packing on dynamic modulus of hot mix asphalt mixtures using threedimensional discrete element method." Constr. Build. Mater., 26, 302-309.

Zhang, J., Li, J., Yao, Y., Zheng, J. and Gu, F. (2018a). "Geometric anisotropy modeling and shear behavior evaluation of graded crushed rocks." Constr. Build. Mater., 183, 346-355.

Zhang, J., Peng, J., Zheng, J. and Yao, Y. (2018b). "Characterisation of stress and moisture-dependent resilient behaviour for compacted clays in South China." Road Mater. Pavement, 1-14. DOI: 10.1080/14680629.2018.1481138

Zhang, J., Peng, J., Li, J. and Zheng, J. (2018c). "Variation of resilient modulus with soil suction for cohesive soils in south China." Int. J. Civil Eng., 1-13. DOI: 10.1007/s40999-018-0315-y

Zhang Y. and Wong L. (2018d). "A review of numerical techniques approaching microstructures of crystalline rocks." Comput. Geosci-UK., 115, 167-187. 